\title{
High- $Q$ terahertz Bragg resonances within a metal parallel plate waveguide
}

\author{
S. Sree Harsha, N. Laman, ${ }^{\text {a) }}$ and D. Grischkowsky ${ }^{\text {b) }}$ \\ School of Electrical and Computer Engineering, Oklahoma State University, Stillwater, \\ Oklahoma 74078, USA
}

(Received 4 February 2009; accepted 13 February 2009; published online 6 March 2009)

\begin{abstract}
One-dimensional (1D) Bragg waveguides are demonstrated at terahertz (THz) frequencies. Lithographically made 1D symmetric and asymmetric dielectric gratings on metallized high conductivity $\mathrm{Si}$ chips are incorporated within metal parallel plate waveguides to form Bragg waveguides. These waveguides have high throughput and have Bragg resonances with linewidths approaching $6 \mathrm{GHz}$ and $Q$ as high as 430 . These high- $Q$ resonant Bragg waveguides are excellent structures for applications in $\mathrm{THz}$ sensing. (C) 2009 American Institute of Physics.
\end{abstract}

[DOI: $10.1063 / 1.3094919]$

Propagation of electromagnetic radiation through periodic media often results in many interesting and potentially useful phenomena. Periodic structures such as photonic crystal bandgap materials and surface plasmon resonators are currently being researched extensively for their potential as frequency filters and high sensitivity sensors. At the same time a great deal of work utilizing waveguides at terahertz frequencies has been done, and the metal parallel plate waveguide (PPWG) has been found to be an excellent guiding structure due its low-loss transverse electromagnetic (TEM) mode propagation. ${ }^{1}$ With its ease of integrating active and passive components, the PPWG has been shown to offer significant potential as a terahertz $(\mathrm{THz})$ interconnect device. ${ }^{2}$ Two-dimensional metallic $\mathrm{THz}$ photonic structures used as the bottom plate of a PPWG, have shown sharp bandgap turn-ons with high dynamic range. ${ }^{3}$ A one-dimensional (1D) $\mathrm{THz}$ photonic metal PPWG with high throughput and well defined stopbands has also been demonstrated. ${ }^{4}$ Periodic deep grooves in one waveguide plate excited higher order propagating and nonpropagating modes, thereby producing broad stopbands with sharp turn-offs and turn-ons. Incorporating a defect produced a sharp $17 \mathrm{~dB}$ transmission resonance with a $Q$ of $120{ }^{4}$

Bragg gratings have long been used as frequency selective devices for integrated optics. Bragg gratings are capable of extremely sharp frequency responses and are commonly used in fiber optics for filtering and in semiconductor lasers as part of cavity resonators. ${ }^{5}$ Optical fiber Bragg grating sensors have been widely used in many sensing applications including temperature, strain, and pressure measurements. ${ }^{6,7}$

In this letter, we describe the fabrication and incorporation of $1 \mu \mathrm{m}$ thick dielectric $\mathrm{THz}$ Bragg gratings within a single TEM mode metal PPWG and experimentally demonstrate the resulting high- $Q$ resonances. High resolution measurements of $\mathrm{THz}$ Bragg resonances of different strengths and linewidths were obtained.

High quality one dimensional gratings were fabricated in a clean room using standard lithographic and metallization techniques. An approximately $1 \mu \mathrm{m}$ thick layer of Shipley S1813 photoresist was spun onto a high conductivity Si wafer which was previously coated with a $300 \mathrm{~nm}$ thick Al

\footnotetext{
${ }^{a)}$ Current address: Zomega THz Corp., 1223 People's Ave., Troy, NY 12180.

${ }^{b)}$ Electronic mail: daniel.grischkowsky@okstate.edu.
}

layer. With a custom designed lithographic mask, the photoresist film was exposed and developed. After the lithography, the soft photoresist gratings on the wafer were baked at $150{ }^{\circ} \mathrm{C}$ in a convection oven, transforming them into permanent, hard polymer structures. The final thickness of the grating structure, measured using an atomic force microscope, was $900 \mathrm{~nm}$. The $0.3 \mathrm{~mm}$ thick wafer was diced into $15 \mathrm{~mm}$ wide $\times 30 \mathrm{~mm}$ long chips to fit the PPWG setup shown in Fig. 1. Two different 1D grating sample chips were fabricated; symmetric sample A with $80 \mu \mathrm{m}$ of photoresist followed by $80 \mu \mathrm{m}$ of space (160 $\mu \mathrm{m}$ period), and asymmetric sample B with $75 \mu \mathrm{m}$ of photoresist followed by $25 \mu \mathrm{m}$ of space (100 $\mu \mathrm{m}$ period). The 1D THz Bragg PPWG was constructed by sandwiching a sample chip between the two polished Al plates of the original PPWG with the metalized face of the chip serving as the $30 \mathrm{~mm}$ long new bottom plate of the waveguide. $50 \mu \mathrm{m}$ spacers separated the top Al plate and the sample chip. Two planocylindrical high resistivity $\mathrm{Si}$ lenses were used to in-couple and out-couple the $\mathrm{THz}$ pulses. ${ }^{1}$

As shown in Fig. 1, the THz pulse propagation was perpendicular to the lines of the grating, with the $\mathrm{THz}$ polarization being perpendicular to the plates. The resulting 1D Bragg waveguide assembly was then placed in the confocal beam waist of a photoconductively switched terahertz time domain spectroscopy (THz-TDS) system ${ }^{8,9}$ for characterization. The average of nine measured $\mathrm{THz}$ output pulses from the symmetric Bragg waveguide (sample A), and the average of six for the asymmetric Bragg waveguide (sample B) are shown in Figs. 2(a) and 3(a). The inset in each figure shows the ringing structure following the main pulse which clearly continues beyond $60 \mathrm{ps}$. The experimentally determined linewidths were obtained from pulse train measurements extending to $150 \mathrm{ps}$, which determines the corresponding spectral resolution to be $6.7 \mathrm{GHz}$. Figures 2(b) and 3(b) show the

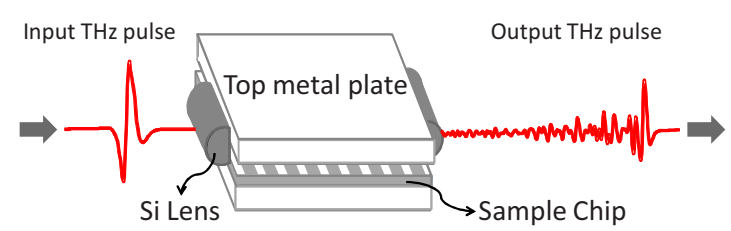

FIG. 1. (Color online) Cross-sectional view of the PPWG assembly together with the sample chip. 

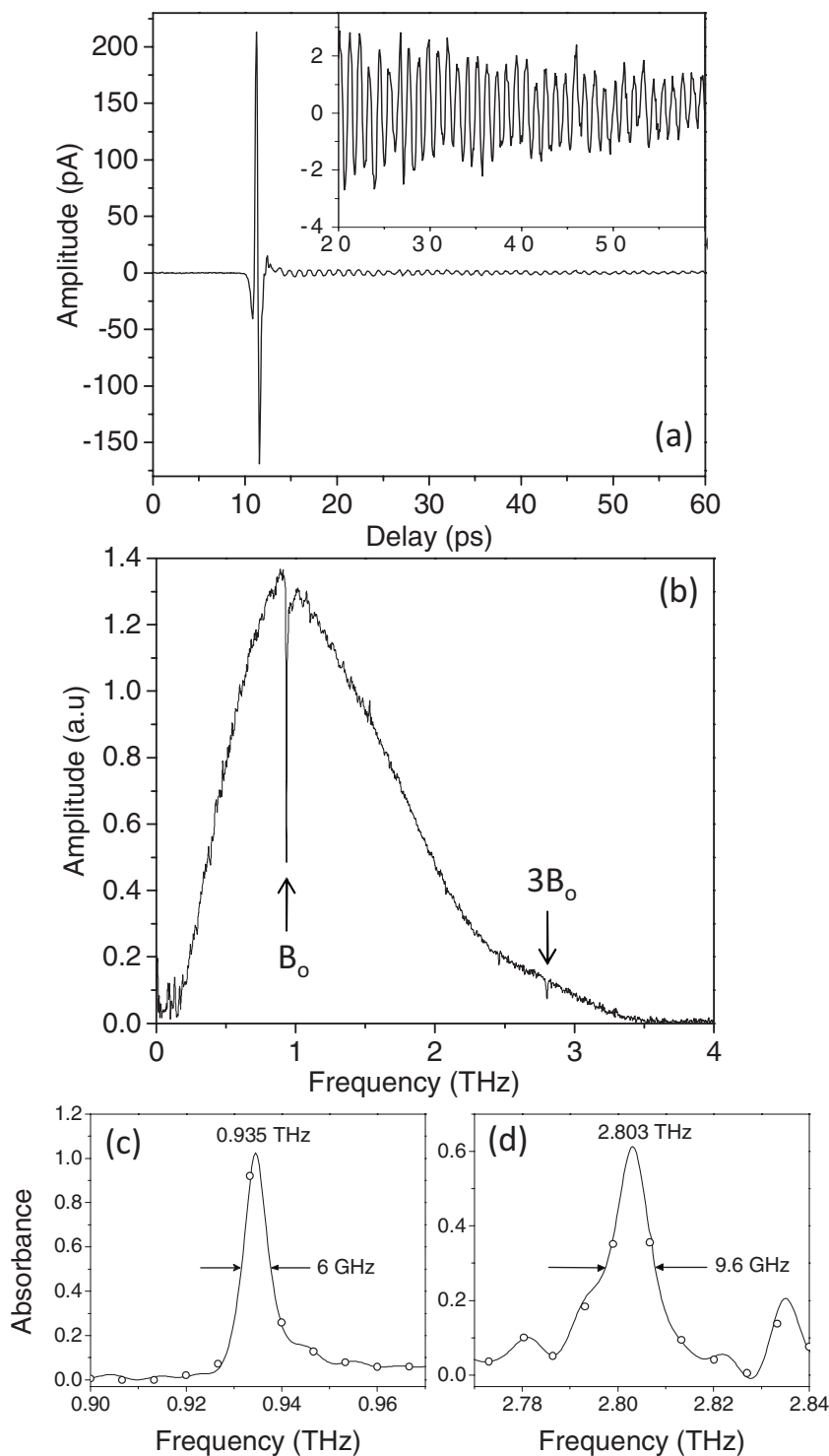

FIG. 2. (a) Output terahertz pulse from type A Bragg waveguide. (b) Transmission spectrum of output pulse. [(c) and (d)] Waveguide absorbance spectrum zoomed in at the fundamental Bragg frequency $B_{o}$ and its third harmonic $3 B_{o}$. Open circles indicate nonzero-padded data points separated by $6.7 \mathrm{GHz}$.

amplitude spectrum of samples A and B, clearly indicating the Bragg resonances (arrows).

The spectra of the transmitted $\mathrm{THz}$ pulses were calculated after zero padding, i.e., the 150 ps data pulse was extended to 2667 ps by adding a train of zeros at the end of the pulse. Zero padding is often used in THz-TDS, whereby extending the time domain data with zeros causes the subsequent Fourier transform to perform an interpolation in frequency domain between the linearly independent real data points, here separated from each other by $6.67 \mathrm{GHz}$, as determined by the 150 ps scan length.

We have determined our line centers from the interpolated amplitude spectrum of the zero-padded average of nine independent transmitted $\mathrm{THz}$ pulse measurements for sample $A$ and six for sample B. The indicated uncertainty of $\pm 0.5 \mathrm{GHz}$ about the fundamental frequency $B_{o}$ $=0.9347 \mathrm{THz}$ for the zero-padded average of sample A, is the spectral range that contains all the central frequency peaks of the nine individual zero-padded terahertz pulses.
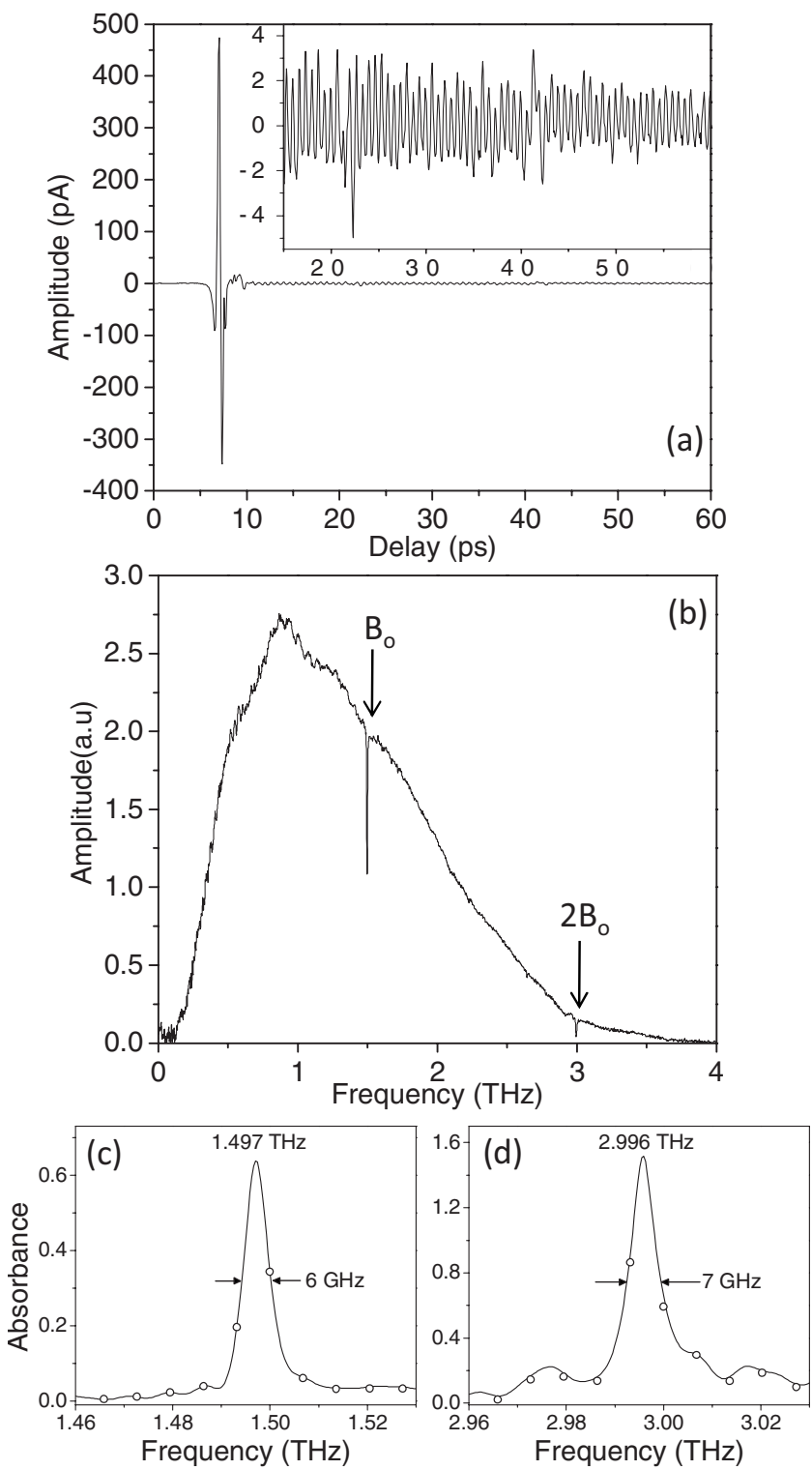

FIG. 3. (a) Output terahertz pulse from type B Bragg waveguide. (b) Transmission spectrum of output pulse. [(c) and (d)] Waveguide absorbance spectrum zoomed in at the fundamental Bragg frequency $B_{o}$ and its second harmonic $2 B_{o}$. Open circles indicate nonzero-padded data points separated by $6.7 \mathrm{GHz}$.

This same procedure was used for the six individual zeropadded pulses of sample B. This uncertainty is strongly dependent on the signal to noise $(\mathrm{S} / \mathrm{N})$ ratio of the spectrum; as the $\mathrm{S} / \mathrm{N}$ ratio increases, the corresponding uncertainty decreases, and vice versa. It is noteworthy that the $B_{o}$ frequencies have been determined to a precision of $\pm 0.5 \mathrm{GHz}$, compared to the spectral resolution of $6.7 \mathrm{GHz}$. Consequently, for sensor applications frequency shifts of $0.5 \mathrm{GHz}$ or more should be observable.

An important measurement consideration is that, a too early truncation of the time domain pulse and starting the zero padding before the pulse has damped out, results in a broadened line shape accompanied by small spectral shifts. However, when a time pulse signal has disappeared into the noise (as in our experiments), subsequent zero padding should not affect the line shape and not produce observable frequency shifts. ${ }^{10}$ 
TABLE I. The Bragg resonance frequencies $v_{0}$ and their uncertainty, absorbance FWHM $\Delta v$, and $Q$ values listed for the two samples.

\begin{tabular}{lccc}
\hline \hline Waveguide & $\begin{array}{c}v_{\mathrm{o}} \\
(\mathrm{THz})\end{array}$ & $\begin{array}{c}\Delta v \\
(\mathrm{GHz})\end{array}$ & $Q=v_{\mathrm{o}} / \Delta v$ \\
\hline Sample A & $0.935 \pm 0.5 \mathrm{GHz}$ & 6.0 & 155 \\
& $2.803 \pm 5.0 \mathrm{GHz}$ & 9.6 & 290 \\
& & & \\
Sample B & $1.497 \pm 0.5 \mathrm{GHz}$ & 6.0 & 250 \\
& $2.996 \pm 2.0 \mathrm{GHz}$ & 7.0 & 430 \\
\hline \hline
\end{tabular}

Figures 2(c) and 2(d) show the waveguide absorption of sample A, at the $B_{o}$ and $3 B_{o}$ Bragg resonances. Figures 3(c) and 3(d) show the Bragg resonances of sample B, where $2 B_{o}$ is allowed by the asymmetric pattern.

Table I lists the observed resonance frequencies and their uncertainty, their full width at half maximum (FWHM) linewidths, and $Q$ values for both samples; $Q=v_{o} / \Delta v$, where $v_{o}$ is the center frequency and $\Delta v$ is the absorbance FWHM of the resonance. The $Q$ of 430 corresponding to the $2 B_{o}$ $=2.996 \mathrm{THz}$ line is the highest experimentally measured $Q$ for a periodic structure incorporated within a metal terahertz PPWG.

Since the depth of the grating within the waveguide is less than $2 \%$ of the waveguide gap, the grating is a very small perturbation within the PPWG. Hence, this Bragg waveguide supports single TEM mode propagation, and consequently the grating within the waveguide can be considered to be a simple thin Bragg stack of alternating regions of two types of hypothetical bulk material with indices of refraction of 1.0057 and 1.0000. For sample A, the Bragg stack equivalent would be alternating $80 \mu \mathrm{m}$ long regions to achieve a total length of $30 \mathrm{~mm}$ and for sample B, it would be alternating layers of $75 \mu \mathrm{m}$ of $n=1.0057$ and $25 \mu \mathrm{m}$ of $n=1.0000$. Propagating through this Bragg stack, the terahertz pulse would be reflected by each interface plane. If the Bragg condition is not satisfied, the reflections from the subsequent interfaces become progressively out of phase and would eventually cancel out. When the Bragg condition is satisfied, the reflections from each interface add constructively in the backward direction to form a back-reflected peak and a corresponding forward absorption peak, both with the same center frequency.

The first-order Bragg condition is given as $B_{o}=c / 2 n \Lambda$, where $c$ is the speed of light, $n$ is the average refractive index and $\Lambda$ is the grating period. The refractive index of baked S1813 photoresist at $1 \mathrm{THz}$ is $1.65 .^{11}$ Since we have a $\sim 900 \mathrm{~nm}$ layer of photoresist and a gap of $50 \mu \mathrm{m}$, the effective refractive index of the region comprising the photoresist within the waveguide can be calculated to be $1.0057 .^{12}$ This gives an average index of 1.0028 and 1.0043 for types A and B Bragg waveguides, respectively. Using this refractive index, $B_{o}$ for $\Lambda=160 \mu \mathrm{m}$ and $100 \mu \mathrm{m}$ is calculated to be 0.934 and $1.492 \mathrm{THz}$. The experimentally determined resonances at 0.935 and $1.497 \mathrm{THz}$ are in good agreement with the predicted values. The intrinsic line widths predicted from the single reflection coherence length of the Bragg waveguide $(60 \mathrm{~mm}=$ twice the waveguide length) is $5 \mathrm{GHz}$ compared to the experimental resolution of $6.7 \mathrm{GHz}$. Hence the $Q$ predicted for the third harmonic resonance of sample B at $2.996 \mathrm{THz}$ is $\sim 600$, whereas the experimentally obtained $Q$ was 430 .

High- $Q$ resonant structures have great potential in sensing applications, which require narrow, easy to detect features. The 1D Bragg waveguides are ideal for such sensing applications, in particular refractive index sensing, which is important for biological and chemical detection of a number of substances. ${ }^{13}$ One such application would be in the detection of antibodies and antigens. ${ }^{14}$ Antibodies have preferential attraction toward antigens. Hence a dielectric grating of antigens formed on the metallized plate can be used to detect trace amounts of antibodies. When this grating is dipped in a solution containing antibodies, the binding of antibodies to antigens changes the effective index of the Bragg waveguide, thereby causing a shift in the Bragg resonance together with a large change in signal strength.

We have demonstrated high- $Q$, resonant transmission minima of 1D terahertz Bragg gratings embedded within a PPWG. $Q$ values as high as 430 were achieved and are the highest achieved to date in periodic structures in metal terahertz waveguides. This ability to selectively reject very narrow parts of the spectrum make them excellent candidates for sensitive trace chemical sensing and filtering applications.

This work was partially supported by the National Science Foundation.

${ }^{1}$ R. Mendis and D. Grischkowsky, Opt. Lett. 26, 846 (2001).

${ }^{2}$ S. Coleman and D. Grischkowsky, Appl. Phys. Lett. 83, 3656 (2003).

${ }^{3}$ A. Bingham, Y. Zhao, and D. Grischkowsky, Appl. Phys. Lett. 87, 051101 (2005).

${ }^{4}$ A. L. Bingham and D. Grischkowsky, Appl. Phys. Lett. 90, 091105 (2007).

${ }^{5}$ C. R. Giles, J. Lightwave Technol. 15, 1391 (1997).

${ }^{6}$ A. D. Kersey, M. A. Davis, H. J. Patrick, M. LeBlac, K. P. Koo, C. G. Askins, M. A. Putnam, and E. J. Friebele, J. Lightwave Technol. 15, 1442 (1997).

${ }^{7}$ A. Iadicicco, A. Cusano, S. Campopiano, A. Cutolo, and M. Giordano, IEEE Sens. J. 5, 1288 (2005).

${ }^{8}$ D. Grischkowsky, S. Keiding, M. van Exter, and Ch. Fattinger, J. Opt. Soc. Am. B 7, 2006 (1990).

${ }^{9}$ M. van Exter and D. Grischkowsky, IEEE Trans. Microwave Theory Tech. 38, 1684 (1990).

${ }^{10}$ J. Cavanagh, W. J. Fairbrother, A. G. Palmer III, N. J. Skelton, and M. Rance, Protein NMR Spectroscopy: Principles and Practice (Academic, New York, 2007).

${ }^{11}$ C. Wood, J. Cunningham, I. C. Hunter, P. Tosch, E. H. Linfield, and A. G Davies, Int. J. Infrared Millim. Waves 27, 557 (2006).

${ }^{12}$ G. Gallot, S. P. Jamison, R. W. McGowan, and D. Grischkowsky, J. Opt. Soc. Am. B 17, 851 (2000).

${ }^{13}$ W. Liang, Y. Huang, Y. Xu, R. K. Lee, and A. Yariv, Appl. Phys. Lett. 86, 151122 (2005).

${ }^{14}$ D. G. Angeley, Proc. SPIE 4958, 1 (2003). 